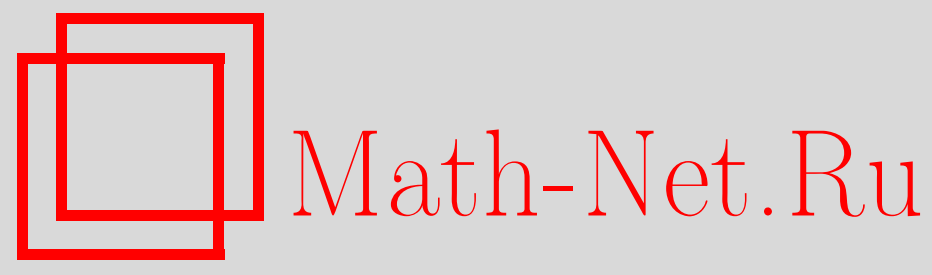

М. Л. Буряков, Асимптотические оценки уровня аффинности для почти всех булевых функций, Дискрет. матем., 2008, том 20, выпуск 3, 73-79

DOI: https://doi.org/10.4213/dm1014

Использование Общероссийского математического портала Math-Net.Ru подразумевает, что вы прочитали и согласны с пользовательским соглашением http://www . mathnet.ru/rus/agreement

Параметры загрузки:

IP: 3.85 .73 .92

26 апреля 2023 г., 14:06:31 


\title{
Асимптотические оценки уровня аффинности для почти всех булевых функций
}

(ㄷ) 2008 г. М. Л. Буряков

\begin{abstract}
Рассматривается асимптотическое поведение параметра булевых функций - уровня аффинности. Показано, что почти все булевы функции от $n$ переменных имеют обобщенный уровень аффинности, превосходящий $n-\alpha \log _{2} n, \alpha>1$, получена асимптотическая верхняя оценка частичного уровня аффинности, рассмотрено асимптотическое поведение уровня аффинности для квадратичных булевых функций.

Работа поддержана Российским фондом фундаментальных исследований, проект 07-01-00154.
\end{abstract}

\section{1. Введение}

Одним из приемов, используемых при исследовании свойств дискретных отображений является изучение их ограничений, в результате чего исходная задача может быть сведена к менее сложной. Примером этого подхода является метод, предложенный в работах $[1,2]$ и использующий параметр булевой функции, называемый уровнем аффинности и характеризующий сложность метода. Исследования свойств уровня аффинности [3, 4] показывают его тесную связь с другими параметрами булевых функций. Кроме того, определенный интерес представляет изучение поведения уровня аффинности асимптотически, подобно поведению некоторых других характеристик (неразложимости почти всех булевых функций [6], тривиальности группы инерции для почти всех булевых функций в полной аффинной группе [7]). Этой проблеме посвящена, в частности, работа [5].

В настоящей работе проводится исследования поведения обобщенного уровня аффинности в целом и получена нижняя оценка обобщенного уровня аффинности для почти всех булевых функций.

\section{2. Основные понятия и определения}

Будем обозначать $\mathbb{F}_{2}$ поле из двух элементов, $V_{n}=\mathbb{F}_{2}^{n}$ - линейное пространство векторстолбцов размерности $n$ над полем $\mathbb{F}_{2}$. Вес Хемминга двоичного вектора $\mathbf{x}=\left(x_{1}, \ldots, x_{n}\right)$ определяется как $\operatorname{wt}(\mathbf{x})=\sum_{i=1}^{n} x_{i}$. Пусть $L-$ некоторое подпространство $V_{n}, \operatorname{dim} L=r$, и $\mathbf{v} \in V_{n}$. Смежный класс $\pi=L \oplus \mathbf{v}$ будем называть плоскостью размерности $r$ пространства $V_{n}$ и писать $\operatorname{dim} \pi=r$. Будем считать, что $\operatorname{dim} \pi=-1$, если $\pi=\varnothing$, и $\operatorname{dim} \pi=0$, если $\pi=\{\mathbf{u}\}, \mathbf{u} \in V_{n}$. Множество всех плоскостей пространства $V_{n}$ (включая пустую плоскость) обозначим через $\mathrm{P}\left(V_{n}\right)$. 
Пусть $\left\{\mathbf{e}_{1}, \mathbf{e}_{2}, \ldots, \mathbf{e}_{n}\right\}$ - канонический базис $V_{n}, \mathbf{e}_{i}=(0, \ldots, 0,1,0, \ldots, 0)^{\top}$, где 1 стоит в $i$-й позиции, $i=1,2, \ldots, n$.

Для любого вектора $\mathbf{x}=\left(x_{1}, \ldots, x_{n}\right)^{\top} \in V_{n}$ справедливо равенство

$$
\mathbf{x}=x_{1} \mathbf{e}_{1} \oplus \ldots \oplus x_{n} \mathbf{e}_{n},
$$

где $\oplus$ обозначает сложение в $\mathbb{F}_{2}$ и покомпонентное сложение векторов в $V_{n}$.

Пусть $n, m$ - натуральные числа. Обозначим через $\mathscr{F}_{n, m}$ множество всех отображений из $V_{n}$ в $V_{m}$. В случае, когда $m=1$, множество $\mathscr{F}_{n}=\mathscr{F}_{n, 1}$ есть множество всех отображений из $V_{n}$ в $\mathbb{F}_{2}$, то есть множество всех булевых функций от $n$ переменных.

Для булевой функции $f$ из $\mathscr{F}_{n}$ ее значение на векторе $\mathbf{x}=x_{1} \mathbf{e}_{1} \oplus \ldots \oplus x_{n} \mathbf{e}_{n}$ будем обозначать

$$
f(\mathbf{x})=f\left(x_{1} \mathbf{e}_{1} \oplus \ldots \oplus x_{n} \mathbf{e}_{n}\right)=f\left(x_{1}, \ldots, x_{n}\right) .
$$

Любая функция из $\mathscr{F}_{n}$ может быть представлена в виде полинома

$$
f\left(x_{1}, \ldots, x_{n}\right)=\bigoplus_{\mathbf{u} \in V_{n}} \lambda_{\mathbf{u}}\left(\prod_{i=1}^{n} x_{i}^{u_{i}}\right), \quad \lambda_{\mathbf{u}} \in \mathbb{F}_{2},
$$

называемого алгебраической нормальной формой (АНФ) этой функции.

Алгебраической степенью функции $f$, обозначаемой $\operatorname{deg}(f)$, является максимальное значение $\operatorname{wt}(\mathbf{u})$ по тем $\mathbf{u}$, для которых $\lambda_{\mathbf{u}} \neq 0$. Через $\operatorname{deg}\left(f, x_{i}\right)$ обозначается максимальное значение $\operatorname{wt}(\mathbf{u})$ по тем $\mathbf{u}$, для которых $\lambda(\mathbf{u}) \neq 0$ и $i$-я компонента вектора и равна 1 (то есть максимальная степень монома, в котором содержится переменная $x_{i}$ ).

Обозначим через $\mathscr{A}_{n, m}$ подмножество аффинных отображений множества $\mathscr{F}_{n, m}$. При этом $\mathscr{A}_{n, 1}=\mathscr{A}_{n}-$ множество аффинных булевых функций от $n$ переменных, то есть функций $f \in \mathscr{F}_{n}$, для которых $\operatorname{deg}(f) \leqslant 1$.

Пусть $f \in \mathscr{F}_{n}$. Для наборов $1 \leqslant i_{1}<\ldots<i_{k} \leqslant n, \mathbf{b}=\left(b_{1}, b_{2}, \ldots, b_{k}\right)^{\top} \in V_{k}$ при $k \leqslant n$ обозначим через $f_{i_{1}, \ldots, i_{k}}^{b_{1}, \ldots, b_{k}}$ булеву функцию из $\mathscr{F}_{n-k}$, полученную из $f$ фиксацией переменных $x_{i_{1}}=b_{1}, \ldots, x_{i_{k}}=b_{k}$ и называемую подфункцией функции $f$.

Булева функция $f$ из $\mathscr{F}_{n}$ называется $k$-аффинной, $0 \leqslant k \leqslant n-1$, если существуют наборы $1 \leqslant i_{1}<\ldots<i_{k} \leqslant n, \mathbf{b}=\left(b_{1}, \ldots, b_{k}\right)^{\top} \in V_{k}$ такие, что $f_{i_{1}, \ldots, i_{k}}^{b_{1}, \ldots, b_{k}}$ является аффинной, то есть $\operatorname{deg}\left(f_{i_{1}, \ldots, i_{k}}^{b_{1}, \ldots, b_{k}}\right) \leqslant 1$.

Уровнем аффинности la $(f)$ булевой функции $f$ из $\mathscr{F}_{n}$ называется минимальное число $k$, для которого $f$ является $k$-аффинной.

Частичным уровнем аффинности $\mathrm{la}^{0}(f)$ булевой функции $f$ из $\mathscr{F}_{n}$ называют минимальное число $k$, для которого существует набор переменных $i_{1}, \ldots, i_{k}$ такой, что $f_{i_{1}, \ldots, i_{k}}^{0, \ldots, 0}$ является аффинной.

Понятие $k$-аффинности и уровня аффинности было введено в [2]. В [5] было показано, что асимптотически при $n \rightarrow \infty$ для почти всех булевых функций $\mathrm{la}(f) \geqslant\lceil n / 2\rceil$.

Пусть $\Phi \in \mathscr{F}_{n, m}$ и $M-$ произвольное подмножество пространства $V_{n}$. Ограничением $\left.\Phi\right|_{M}$ отображения $\Phi$ на множество $M$ будем называть отображение $\Phi^{\prime}: M \rightarrow V_{m}$ такое, что $\Phi^{\prime}(\mathbf{x})=\left.\Phi\right|_{M}(\mathbf{x})=\Phi(\mathbf{x})$ для всех $\mathbf{x} \in M$.

Пусть $\Phi \in \mathscr{F}_{n, m}$. Плоскость $\pi \in \mathrm{P}\left(V_{n}\right) \backslash \varnothing$ называется локальной аффинностью булева отображения $\Phi$, если существует аффинное отображение $\Psi \in \mathcal{A}_{n, m}$ такое, что $\left.\Phi\right|_{\pi}=\left.\Psi\right|_{\pi}$.

Обозначим

$$
\widetilde{\mathrm{P}}_{\Phi}\left(V_{n}\right)=\left\{\pi \in \mathrm{P}\left(V_{n}\right) \backslash \varnothing\left|\exists \Psi \in \mathscr{A}_{n, m}: \Phi\right|_{\pi}=\left.\Psi\right|_{\pi}\right\}
$$


совокупность локальных аффинностей отображения $\Phi$.

Обобщенным уровнем аффинности отображения $\Phi \in \mathscr{F}_{n, m}$ называется неотрицательное целое число

$$
\mathfrak{R a}(\Phi)=n-\max _{\pi \in \widetilde{\mathrm{P}}_{\Phi}\left(V_{n}\right)} \operatorname{dim} \pi
$$

Замечание 1. Значение обобщенного уровня аффинности, в отличие от обычного уровня аффинности, является аффинным инвариантом, то есть инвариантом относительно действия на функцию полной аффинной группы (см. [6]).

Замечание 2. Очевидно, что

$$
\mathfrak{L a}(f) \leqslant \mathrm{la}(f) \leqslant \mathrm{la}^{0}(f)
$$

для произвольной булевой функции $f$.

\section{3. Основные результаты}

Вначале докажем асимптотическую верхнюю оценку для обобщенного уровня аффинности булевых функций.

Теорема 1. Пусть $\alpha \in \mathbf{R}, \alpha>1,-$ произвольная фиксированная константа. Тогда асимптотически при $n \rightarrow \infty$ для почти всех функции из $\mathscr{F}_{n}$

$$
\mathfrak{R a}(f) \geqslant n-\alpha \log _{2} n \text {. }
$$

Доказательство. Известно (см. [8]), что число $r$-мерных плоскостей пространства $V_{n}$ равно $2^{n-r}\left[\begin{array}{l}n \\ r\end{array}\right]$, где

$$
\left[\begin{array}{l}
n \\
r
\end{array}\right]=\frac{\left(2^{n}-1\right)\left(2^{n}-2\right) \ldots\left(2^{n}-2^{r-1}\right)}{\left(2^{r}-1\right)\left(2^{r}-2\right) \ldots\left(2^{r}-2^{r-1}\right)}, \quad 1 \leqslant r \leqslant n, \quad\left[\begin{array}{c}
n \\
0
\end{array}\right]=1 .
$$

Рассмотрим какую-либо $r$-мерную плоскость $L$ пространства $V_{n}$. Пусть $a_{L}-$ число булевых функций из $\mathscr{F}_{n}$, ограничение которых на $L$ есть аффинная функция. Нетрудно понять, что это число не зависит от выбора $L$, а зависит только от числа $r$. Тогда число булевых функций $f \in \mathscr{F}_{n}$, для которых $\mathfrak{L a}(f) \leqslant n-r$, будет не больше, чем $2^{n-r}\left[\begin{array}{l}n \\ r\end{array}\right] a_{L}$.

Рассмотрим теперь конкретную $r$-мерную плоскость вида $\mathbb{F}_{2}^{r} \times\{(0, \ldots, 0)\}$. Ограничение функции $f$ на эту плоскость является аффинным тогда и только тогда, когда $f$ не содержит мономы длины $2,3, \ldots, r$, состоящие только из переменных $x_{1}, \ldots, x_{r}$. Число функций, обладающих таким свойством, равно

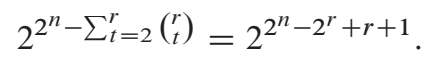

Значит, $a_{L}=2^{2^{n}-2^{r}+r+1}$. Таким образом, с учетом (1) получаем, что число функций, имеющих аффинное ограничение на $r$-мерных плоскостях, не больше, чем

$$
2^{n-r}\left[\begin{array}{l}
n \\
r
\end{array}\right] 2^{2^{n}-2^{r}+r+1}
$$


а их доля относительно всех функций из $\mathscr{F}_{n}$ равна

$$
2^{n-r}\left[\begin{array}{l}
n \\
r
\end{array}\right] 2^{-2^{r}+r+1}
$$

Далее, поскольку каждый множитель в числителе дроби, определяющей $\left[\begin{array}{l}n \\ r\end{array}\right]$, меньше $2^{n}$, а каждый множитель в знаменателе больше или равен $2^{r-1}$, справедливо неравенство

$$
\left[\begin{array}{l}
n \\
r
\end{array}\right]<2^{n(r-1)-(r-1)^{2}}=2^{n(r-1)-r^{2}+2 r-1} .
$$

Отсюда следует, что доля функций, имеющих аффинное ограничение на $r$-мерных плоскостях, меньше, чем

$$
2^{n(r-1)-r^{2}+2 r-1-2^{r}+r+1}=2^{n(r-1)-r^{2}+3 r-2^{r}} .
$$

Положим теперь

$$
r=\alpha \log _{2} n, \quad \alpha \in \mathbf{R}, \quad \alpha>1,
$$

и устремим $n \rightarrow \infty$. Получим, что доля функций, имеющих аффинное ограничение на плоскостях размерности, большей $\alpha \log _{2} n$, стремится к 0. Иными словами, почти все булевы функции от $n$ переменных имеют аффинное ограничение на плоскостях размерности, меньшей $\alpha \log _{2} n$. Очевидно, что если функция аффинна на плоскости размерности $r>0$, то она также аффинна на некоторой плоскости размерности $r-1$. Отсюда непосредственно следует утверждение теоремы.

Так как $\mathfrak{L a}(f) \leqslant 1 \mathrm{a}(f)$, из теоремы 1 , как следствие, можно получить следующий результат.

Следствие 1. Асимптотически при $n \rightarrow \infty$ почти все булевы функции из $\mathscr{F}_{n}$ имеют уровень аффинности, превосходящий $n-\alpha \log _{2} n, \alpha \in \mathbf{R}, \alpha>1$.

Заметим, что аналогичное утверждение верно и для частичного уровня аффинности $1 \mathrm{a}^{0}$.

Теорема 2. Пусть $\xi_{n}-$ случайная величина, распределенная равномерно на множестве $\mathscr{F}_{n}, \alpha \in \mathbf{R}, \alpha>1 u$

$$
P_{n}=\mathbf{P}\left\{\mathfrak{L a}\left(\xi_{n}\right)>n-\alpha \log _{2} n\right\} .
$$

Тогда

$$
\lim _{n \rightarrow \infty} P_{n}=1 .
$$

Эта теорема вытекает из теоремы 1 .

Рассмотрим теперь вопрос о верхней границе уровня аффинности. Пусть $f \in \mathscr{F}_{n}$. Обозначим $\operatorname{len}^{*}(f)$ число нелинейных мономов в АНФ функции $f, n^{*}$ - число переменных $x_{i}$, для которых $\operatorname{deg}\left(f, x_{i}\right)>1, i=1, \ldots, n$.

Пусть также

$$
\mathscr{F}_{n} \psi(n)=\left\{f \in \mathscr{F}_{n}: \operatorname{len}^{*}(f)<\frac{2^{n}}{\psi(n)}\right\},
$$

где $\psi(n)-$ некоторая функция, $\psi(n) \neq 0$. 
Теорема 3. Пусть $\varphi(n)-$ произвольная монотонная неограниченная функция, $\varphi(n)>0$ для любого $n ; \beta \in \mathbf{R}, \beta>1$ - некоторая фиксированная константа. Тогда асимптотически при $n \rightarrow \infty$ для почти всех функииии из $\mathscr{F}_{n}^{\varphi^{\beta}}(n)$

$$
\operatorname{la}^{0}(f) \leqslant n-\log _{2} \varphi(n) .
$$

Доказательство. Для произвольной булевой функции $f \in \mathscr{F}_{n}$ составим двоичную матрицу $M_{f}=\left\|M_{f}\langle i, j\rangle\right\|$ из $\operatorname{len}^{*}(f)=s$ строк и $n^{*}=p$ столбцов, полагая $M_{f}\langle i, j\rangle=1$, если в $i$-м мономе функции $f$ содержится переменная $x_{j}$, и $M_{f}\langle i, j\rangle=0$ в противном случае (здесь мы считаем, что мономы каким-либо образом упорядочены, например, в лексикографическом порядке).

Понятно, что различным функциям из $\mathscr{F}_{n}$ соответствуют различные матрицы $M_{f}$, если рассматривать эти функции с точностью до аффинных частей.

Будем говорить, что подмножество $P$ столбцов матрицы $M_{f}$ является аннулирующим, если для любой $i$-й строки, $i=1, \ldots, s$, этой матрицы существует $k$-й столбец, принадлежащий множеству $P$, такой, что $M_{f}\langle i, k\rangle=1$.

Легко понять, что $\mathrm{la}^{0}(f)$ равен минимальной мощности аннулирующего множества для матрицы $M_{f}$.

Рассмотрим теперь случай, когда первые $t$ столбцов матрицы $M_{f}$ образуют аннулирующее множество. Очевидно, что число матриц, обладающих таким свойством, равно $\left(2^{t}-1\right)^{s} 2^{(p-t) s}$, а их доля по отношению ко всем двоичным матрицам размера $s \times p$ равна

$$
\left(2^{t}-1\right)^{s} 2^{(p-t) s} / 2^{p s}=\left(1-\frac{1}{2^{t}}\right)^{s} .
$$

Положим $t=n-\log _{2} \varphi(n), s=2^{n} / \varphi^{\beta}(n)$, где $\varphi(n)$ и $\beta$ удовлетворяют условиям теоремы.

При таком выборе параметров $t$ и $s$ можно считать, что рассматриваются только функции из множества $\mathscr{F}_{n}^{\varphi^{\beta}(n)}$. Кроме этого получаем, что доля функций, для которых частичный уровень аффинности меньше либо равен $t=n-\log _{2} \varphi(n)$, больше или равна

$$
\left(1-\frac{1}{2^{n-\log _{2} \varphi(n)}}\right)^{2^{n} / \varphi^{\beta}(n)}=\left(\left(1-\frac{\varphi(n)}{2^{n}}\right)^{-2^{n} / \varphi(n)}\right)^{-1 / \varphi^{\beta-1}(n)},
$$

то есть стремится к 1 при $n \rightarrow \infty$. Отсюда следует утверждение теоремы.

Поскольку

$$
\mathfrak{L a}(f) \leqslant \mathrm{la}(f) \leqslant \mathrm{la}^{0}(f),
$$

из теоремы 3 непосредственно получаем следующее утверждение.

Следствие 2. Если $\varphi(n)$ и $\beta$ удовлетворяют условию теоремы 3, то асимптотически при $n \rightarrow \infty$ почти все булевы функиии из $\mathscr{F}_{n}^{\varphi^{\beta}(n)}$ имеют уровень аффинности, не превосходящий $n-\log _{2} \varphi(n)$. Аналогичное утверждение верно и для обобщенного уровня аффинности.

Если рассматривать класс функций с ограничением на их степень, то из теоремы 3 получаем следующее утверждение. 
Следствие 3. Пусть $\beta \in \mathbf{R}, \beta>1$, - произвольная фиксированная константа. Тогда асимптотически при $n \rightarrow \infty$ для почти всех функций $f \in \mathscr{F}_{n}$ таких, что $\operatorname{deg}(f) \leqslant d$, где d фиксировано,

$$
\mathrm{la}^{0}(f) \leqslant \frac{\beta-1}{\beta} n+\frac{1}{\beta} \log _{2} D_{d}^{n},
$$

где

$$
D_{d}^{n}=\sum_{i=0}^{d}\left(\begin{array}{l}
n \\
i
\end{array}\right)
$$

Доказательство. Если $f \in \mathscr{F}_{n}$ и $\operatorname{deg}(f) \leqslant d$, то в АНФ функции $f$ содержатся мономы степени не выше $d$, и их число $\operatorname{len}(f) \leqslant D_{d}^{n}$. Тогда в теореме 3 можно положить $\varphi(n)=$ $\left(2^{n} / D_{d}^{n}\right)^{1 / \beta}$, откуда

$$
\mathrm{la}^{0}(f) \leqslant n-\frac{1}{\beta} \log _{2}\left(\frac{2^{n}}{D_{d}^{n}}\right)=\frac{\beta-1}{\beta} n+\frac{1}{\beta} \log _{2} D_{d}^{n}
$$

для почти всех $f$ при $n \rightarrow \infty$.

Замечание 3. Нетрудно видеть, что нижняя и верхняя асимптотические границы уровня аффинности очень похожи, поэтому представляет определенный интерес вопрос получения асимптотического равенства или по крайней мере сужения разницы между верхними и нижними оценками.

В заключение рассмотрим вопрос о распределении уровня аффинности для квадратичных булевых функций. Эти функции интересны, в частности, тем, что именно на них достигается максимальное значение уровня аффинности (см. [3]). Заметим также, что для любой квадратичной булевой функции $f$, очевидно, $1 \mathrm{a}(f)=\mathrm{la}^{0}(f)$.

Каждой квадратичной булевой функции $f$ можно поставить в соответствие неориентированный граф $G_{f}(V, E)$, где множество $V$ совпадает с множеством переменных функции $f$, а ребро $\left(x_{i}, x_{j}\right)$ содержится в множестве $E$ тогда и только тогда, когда АНФ функции $f$ содержит моном $x_{i} x_{j}$. Если в АНФ функции $f$ содержится моном $x_{k}$, то можно считать, что в графе $G_{f}$ есть петля $x_{k} x_{k}$. Заметим, однако, что наличие и вид аффинной части у булевой функции не влияет на ход дальнейших рассуждений.

Очевидно, что если граф $G_{f}$ имеет клику (то есть полный подграф) мощности $d$, то $\mathrm{la}(f) \geqslant d-1$ (см. [2]). Если $d_{\max }\left(G_{f}\right)-$ мощность максимальной клики для графа $G_{f}$, To la $(f)=d_{\max }\left(G_{f}\right)-1$.

В [9] показано, что вероятность того, что для случайного графа мощность максимальной клики $d_{\max }(G)$ удовлетворяет неравенствам

$$
\lfloor M(n)\rfloor \leqslant d_{\max }(G) \leqslant\lceil M(n)\rceil,
$$

стремится к 1 при $n \rightarrow \infty$. Здесь

$$
M(n)=2\left(\log _{2} n-\log _{2} \log _{2} n+\log _{2} \frac{e}{2}\right)+1 .
$$

С учетом этого результата и предыдущих рассуждений можно сформулировать следующее утверждение. 
Теорема 4. Вероятность того, что для случайной булевой функции $f \in \mathscr{F}_{n}, \operatorname{deg}(f) \leqslant 2$, справедливы неравенства

$$
\lfloor M(n)\rfloor \leqslant \operatorname{la}(f) \leqslant\lceil M(n)\rceil,
$$

где

$$
M(n)=2\left(\log _{2} n-\log _{2} \log _{2} n+\log _{2} \frac{e}{2}\right)
$$

стремится $\kappa 1$ при $n \rightarrow \infty$.

\section{Список литературы}

1. Логачев О. А., Сальников А. А., Ященко В. В., Корреляционная иммунность и реальная секретность. В сб.: Математика и безопасность информационных технологий. МЦНМО, Москва, 2004 , c. $165-170$.

2. Логачев О. А., Сальников А. А., Ященко В. В., Комбинирующие $k$-аффинные функции. В сб.: Математика и безопасность информационных технологий. МЦНМО, Москва, 2004, с. 176-178.

3. Буряков М. Л., Логачев О. А., Об уровне аффинности булевых функций. Дискретная математика (2005) 17, №4, 98-107.

4. Буряков М. Л., О связи уровня аффинности с криптографическими параметрами булевых функций. Дискретная математика (2008) 20, №2, 3-14.

5. Буряков М. Л., Логачев О. А., О распределении уровня аффинности на множестве булевых функций. В сб.: Математика и безопасность информационных технологий. МЦНМО, Москва, 2005, c. 141-146.

6. Логачев О. А., Сальников А. А., Ященко В. В., Булевы функиии в теории кодировании и криптологии. МЦНМО, Москва, 2004.

7. Шеннон К., Синтез двухполюсных переключательных схем. В сб.: Работы по теории информации и кибернетике. ИЛ, Москва, 1963, с. 59-105.

8. Мак-Вильямс Ф. Дж., Слоэн Н. Дж. А., Теория кодов, исправляющих ошибки. Связь, Москва, 1979.

9. Matula D. W., The largest clique size in random graph, Techn. Rep. CS 7608. Dept. Comput. Sci., Southern Methodist Univ., 1976.

Статья поступила 10.06.2008. 